\title{
Enabling remote learning system for virtual personalized preferences during COVID-19 pandemic
}

\author{
Sadia Ali ${ }^{1}$ - Yaser Hafeez ${ }^{1}$ - Muhammad Azeem Abbas ${ }^{1}$ • Muhammad Aqib ${ }^{1}$. \\ Asif Nawaz ${ }^{1}$
}

Received: 30 June 2020 / Revised: 18 June 2021 / Accepted: 2 August 2021/

Published online: 17 August 2021

(C) The Author(s), under exclusive licence to Springer Science+Business Media, LLC, part of Springer Nature 2021

\begin{abstract}
The education system worldwide has been affected by the Corona Virus Diseases 2019 (COVID-19) pandemic, resulting in the interruption of all educational institutions. Moreover, as a precautionary measure, the lockdown has been imposed that has severely affected the learning processes, especially assessment activities, including exams and viva. In such challenging situations, E-learning platforms could play a vital role in conducting seamless academic activities. In spite of all the advantages of remote learning systems, many hurdles and obstacles, like a selection of suitable learning resources/material encounter by individual users based on their interests or requirements. Especially those who are not well familiar with the internet technology in developing countries and are in need of a platform that could help them in resolving the issues related to the online virtual environment. Therefore, in this work, we have proposed a mechanism that intelligently and correctly predicts the appropriate preferences for the selection of resources relevant to a specific user by considering the capabilities of diverse perspectives users to provide quality online education and to make work from home policy more effective and progressive during the pandemic. The proposed system helps teachers in providing quality online education, familiarizing them with advanced technology in the online environment. It also semantically predicts the preferences for virtual assistance of those users who are in need of learning the new tools and technologies in short time as per their institutional requirements in order to meet the quality standards of online education. The experimental and statistical results have demonstrated that the proposed virtual personalized preferences system has improved overall academic activities as compared to the current method. The proposed system enhanced user's learning abilities and facilitated them in selecting short courses while using different online education tools adopted/suggested by the institutions to conduct online classes/seminars/ webinars etc., as compared to the conventional classes/activities.
\end{abstract}

Keywords Distance education - COVID-19 - Augmented and virtual reality · Recommendation system $\cdot$ Teaching/learning strategies $\cdot$ Architectures for educational technology system $\cdot$ Text mining

Sadia Ali

sadiaalief@gmail.com

Extended author information available on the last page of the article 


\section{Introduction}

Attempts to halt COVID-19 spread via non-pharmaceutical involvements and precautionary measures, for example, self-segregation and societal exclusion, have encouraged a global conclusion of the scholarly world in more than 100 countries [37]. Previous episodes of irresistible sicknesses have provoked far-reaching school closings around the globe, with fluctuating degrees of success $[9,37]$. Calculated displaying has demonstrated that transmission of a flare-up might be deferred by shutting the scholarly community. In any case, adequacy relies upon the contacts youngsters keep up outside of study conditions $[8,37,43]$ that might be successful when sanctioned speedily and impact severely the global education system. This tragic situation caused the closure of all the educational institutions on a temporary basis worldwide. It decreased the morale of students and teachers about good quality of education for better knowledge and success in their careers. Many institutions, including schools, universities, and colleges that provide on-campus education, were closed, and some of them were shifted to remote education due to this challenging situation overnight.

The online learning system shares knowledge globally using web applications and remote tools for conducting education services [3, 12, 20, 25-27, 35]. In an eLearning environment, sharing knowledge requires an internet facility for both learners or teachers located dispersedly to study or teach anytime, anywhere by using advanced skills and technology $[3,7,13,36]$. It is challenging to provide essential skills, knowledge, and advancement in curriculums and technology through traditional or on-campus education, especially in undeveloped areas. Where there is a lack of internet access and limited and inexperienced $[14,30]$ resources. Therefore, the main challenges are being faced by these institutions in the COVID-19 crisis to accept change and adopt advanced technology for education. These institutions have few technological options for conducting online classes and educational activities under limited budgets and resources without compromising the education standards. Thus, institutions, especially universities, shifted their education system from on campus to digitalized according to dire requirements of the current pandemic situation.

Consequently, education quality through eLearning is crucial due to overnight shifting from campus education to online education. The reasons are that students have to learn and arrange advanced technology in the shortest time to come up with current scenario requirements without compromising their education quality and requirements [16, 39]. Similarly, most teachers also have lack knowledge and skills about advanced technologies that are used in online education. Therefore, both students and teachers require a substantial amount of training and arrangements of resources required for online education. Hence, the issues are not that how to provide quality education; it is how educational institutions handle a massive number of students in online education? Because institutions will be assessed on their ability to adapt to changes in technology and maintain the quality of the educational contents. It is difficult to shift all staff, teachers, and students to online classes with their syllabus online overnight due to dispersed locations, and personalized preferences for learning and teaching [7, 12, 14]. Google and Microsoft products such as google classroom, Gmail, Duo, google forms, and Microsoft Team have helped the institutions shift from on-campus to online education. The major problems include dealing with different online advanced technologies for education, different errors during downloading, installation, internet speed, accessibility, video, and audio. This makes the environment less engaging, confusing, and boring for students, and they find it difficult to pay 
proper attention on the topic. Also, the individual interaction of students with the teachers becomes a prominent issue during online education. The burden of teachers increases while demonstration during practical subject using advanced technology. Both teachers and students need to learn about these modern technologies. Therefore, with the adaption and searching of new technologies and resources for online education, there is a need to guide and train students and teachers to enhance their skills and knowledge about online learning methods $[7,14,15]$. Students and teachers required personalized preferences about digital literacy $[5,35,36,39]$. This will help them in selecting the right technology and skill set, which are the prerequisite to use advanced technology.

In light of the issues mentioned earlier, there is a need of a system that could provide personalized options for online education by advising both learners and teachers on how to study and teach according to their specific set of preferences $[6,30]$. Whether preferences relevant to regular courses or relevant to short courses for maintaining quality and standards of education by enhancing their skills and useful with current modern technology available for online education according to their benefits. Therefore, most of the predictions are based on collaborative-based filtering $(\mathrm{CBF})$ and content filtering $(\mathrm{CF})$ to use previous user experiences and current user information, respectively. To help learners for identification of relevant material and experiences according to their viewpoints, feedbacks, and experiences. Therefore, personalized preference predictions are based on former and current user information and rating $[1,7,14,15]$. Hence, a problem in personalized preferences prediction is semantic analysis and term mismatch in preferences [14, 23, 39]. Another major issue is virtual assistance $24 / 7$ for learners to guide them and resolve their issues.

In accordance with the current crisis and personalize issue mitigation, we proposed a virtual remote learning system for personalized preferences predictions using hybrid filtering (HF). $\mathrm{HF}$ is the combination of $\mathrm{CBF}$ and $\mathrm{CF}$ for correct semantic analysis and term mismatch issues resolution. For this, we developed a web-based prediction system for improving personalized preferences using virtual assistance. The proposed system is designed to predict relevant preferences of multi-user perspectives based on semantic analysis to help the user accurately select contents and courses to enhance their skill and knowledge.

\subsection{Research contribution}

Major contributions of the present study are to resolve personalized preferences according to student/ teacher request during online education issues are as follow:

1. In this study, we identified that online education lacks the personalized preferences of learners and teachers virtually. Therefore, to handle virtual personalized preferences semantically, there is a need to accurately predict personalized preferences in multiple perspectives according to their skills and experiences.

2. The proposed system predicts personalized preferences based on semantic analysis using text mining techniques and hybrid filtering. First, the student and teacher preferences on demand are analyzed by the virtual agent semantically using text mining. Then predict a list of personalized preferences by analyzing previous and current similar students, or teachers preferences consist of knowledge, experience, and skill. The list of predictions classified according to the rating of current and previous students' or teachers' feedback. 
3. Later, an empirical evaluation was performed for the verification of the proposed system. A real scenario was used to follow that justifies the validation of the proposed system. As a result, our proposed system outperformed as compared to others without a personalized prediction method.

4. The present study provides a pathway for future practices and research work that provides overview and experimental proof for dealing with pandemic situations in the education field using information technology benefits.

The remaining sections of this work are organized as follows. Section 2 examines work related to this topic. Section 3 contains a detailed description of the proposed system. Section 4 contains the findings of the empirical evaluation and a discussion of the planned system effectiveness investigation in the current pandemic situation. Section 5 concludes the overall research work and makes some recommendations for future work.

\section{Related work}

A significant quantity of literature has been published on detecting the benefits of online education that helps in getting skills and experience during online distance education. Also, many studies describe the need, role, and recent trends in online education for improving the recent challenges of online education.

Consequently, many researchers have argued that a preferential recommendation is an important element of online education in a virtualized environment [14, 31]. To automatically improve services for teaching and counseling with personalized preferences, online learning has become more important in industry and research with virtualized assistance [7, 31]. As a result, researchers have looked into a variety of issues in online learning, such as tutor-student communication [7, 14]. Using accurate mining and analysis processes, students' source selection preferences, student tests/quizzes, and examinations, among other things, are used to recover the linked preferences with online assistance [23, $30,31]$. The majority of existing solutions focus on anticipating a collection of subjects using a recommendation engine based on previous user data. However, due to a lack of adequate information retrieval, they fail in the case of current and new users, and the semantic relevance of the information for multi-perspective users' changes. By adopting online learning to learners' and teachers' experience, capabilities, and requirements, the current study has improved learners' and teachers' online learning performance.

The author in [19] investigated the use of a mobile learning platform in conjunction with an online system to merge digital and real-world circumstances. It can help students identify flaws in order to stimulate their interest in known material. Similarly, data mining is used in a study by [16] to discover the gap between E-learning inappropriate and improper course combination recommendations. The data demonstrated that the proposed method was useful for selecting and extracting course material for skill development when compared to the Model system. Data mining processes like clustering, classification, and mining association rule are used to recommend a large number of user preferences in order to improve learner skills based on their interests [22]. Reusing information to improve recommendations in e-learning [7] looks into several platforms and the function of ecosystems in knowledge exchange in virtually aid learning. When dealing with huge data sets and scalable learners, using chronological data for recommendations can be beneficial [14]. Simultaneously, improve the competency 
performance of online learners by recommending material based on previous similar student performance levels and using visual analytics for online help [12, 23].

Virtual environment (VE) training resembles a genuine situation and allows students to enter the VE to complete tasks [5, 17, 31]. VE also employs 2D (two-dimensional) and 3D wholly immersed interfaces [31]. VE training can be used as a stand-alone training strategy to cope with multi-user views, necessitating the reuse of previous similar ratings as well as current prior preferences/interests of intelligent semantic recommendations via data mining [5, 6, 39]. In [30], the author proposed OntoSIDES, which is at the heart of ontology-based learning systems in educational content. It kept track of the children's actions in order to help them establish their semantic associations.. To improve information prediction, there is a need to reduce irrelevancy and redundancy intelligently during information classification [6, 23, 34]. The problem of an accurate selection of preferences, virtual assistance, multi perspectives, students/teacher's performance, extraction of relevant contents, and maintaining education standard after COVID-19 crisis and situation. The dilemma COVID-19 had a significant influence on the educational structure and shifted from on-campus to remote education. The new structure of education has impacted and changed student and instructor behavior. Hence, in current situations, more appropriate solutions and appropriate technology adoption and selection are difficult to maintain higher satisfaction $[8,9,37,43]$ and education quality $[4,8,38]$.

As a result, a thorough examination of existing systems revealed several flaws, prompting us to propose a method to address these flaws in existing systems during pandemic emergencies. For example, no semantic information extraction [10, 18, 23] suffers from vagueness and misconception of viewpoints of diverse-perspective problem investigation $[2,16]$, both of which demotivate teachers and students to seek solutions and improve their knowledge. Furthermore, without virtual aid, ambiguity, incompleteness, and redundancy in course descriptions can enhance cooperation with relevant staff and performance concerns [5, 31]. As a result, an easy and efficient system is required to decrease complexity and effort, as well as to provide suitable preferences to minimize rigidity in course content and skills [7, $10,16,23]$. The proposed system thus offers full guidance to train users by using various preferences to reduce the problem during online education against each application.

\section{Proposed system}

In this part, we will discuss the intelligent proposed remote learning system developed to address the concerns of virtual personalized preferences during online education. Concerns that have been addressed in this approach were discovered in the literature while conducting this study. Especially after Covid-19 academic institutions all over the globe shifted from on-campus to the remote online learning systems. For remote learning, different platforms like Google classroom, Zoom, institution-specific learning management systems, etc., have been adopted by the institutions with the use of internet technology. To avoid the loss of education during the lockdown situation, institutions have no other option but to move to these tools. But due to certain reasons, they were not able to provide quality education to their students after adopting different platforms for remote education. One main reason for this problem was that some instructors and students were not familiar with these technologies, and it is difficult for them to adopt these platforms for education purposes. Therefore, they search for tutorials and related material to enhance 
their skills to make remote learning fruitful, successful, and better as compared to oncampus education. Therefore, the objective of the proposed system is to assist students and teachers virtually according to their requirements for remote learning. The students during online education face several issues such as uploading assignments, solving quizzes, and downloading course contents. At the same time, on the other side, the teachers tend to deliver lectures, take quizzes and provide content to students due to their distributed location. The teachers and students have to take help from different social media forums for better and high standard education but still cannot get accurate and proper solutions to their problems that lead to wastage of time and resources. Some teachers and students who are familiar with advanced technology also need to follow short courses through online training to enhance their skills and perform their online jobs during a current pandemic situation. Therefore, the proposed system personalized the preferences and predict solutions according to the requirements and interests of the students and the teachers. Thus, the proposed system helped those who are new to the advanced technologies and those who like to sharpen their scales to compete in a modern technological era. The proposed system complete overview is described in Fig. 1.

The system helps teachers and students in meeting each other according to their personalized preferences in a crisis. Virtually, teachers and students resolve their issues using appropriate software for online education, solution problems during online education, and appropriate selection of short courses for skill enhancement. The proposed system's main steps consist of two main phases, i.e., Input collection and the prediction process.

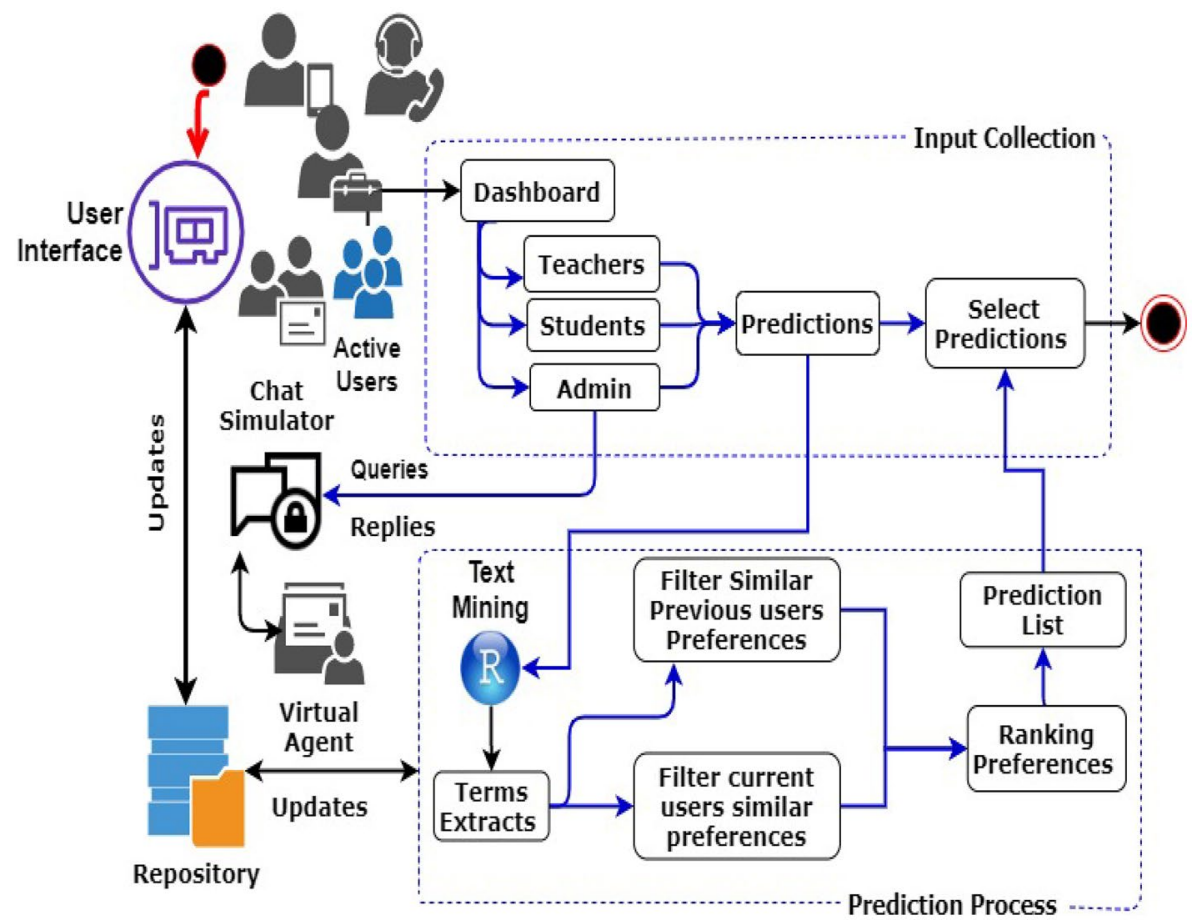

Fig. 1 Proposed System 


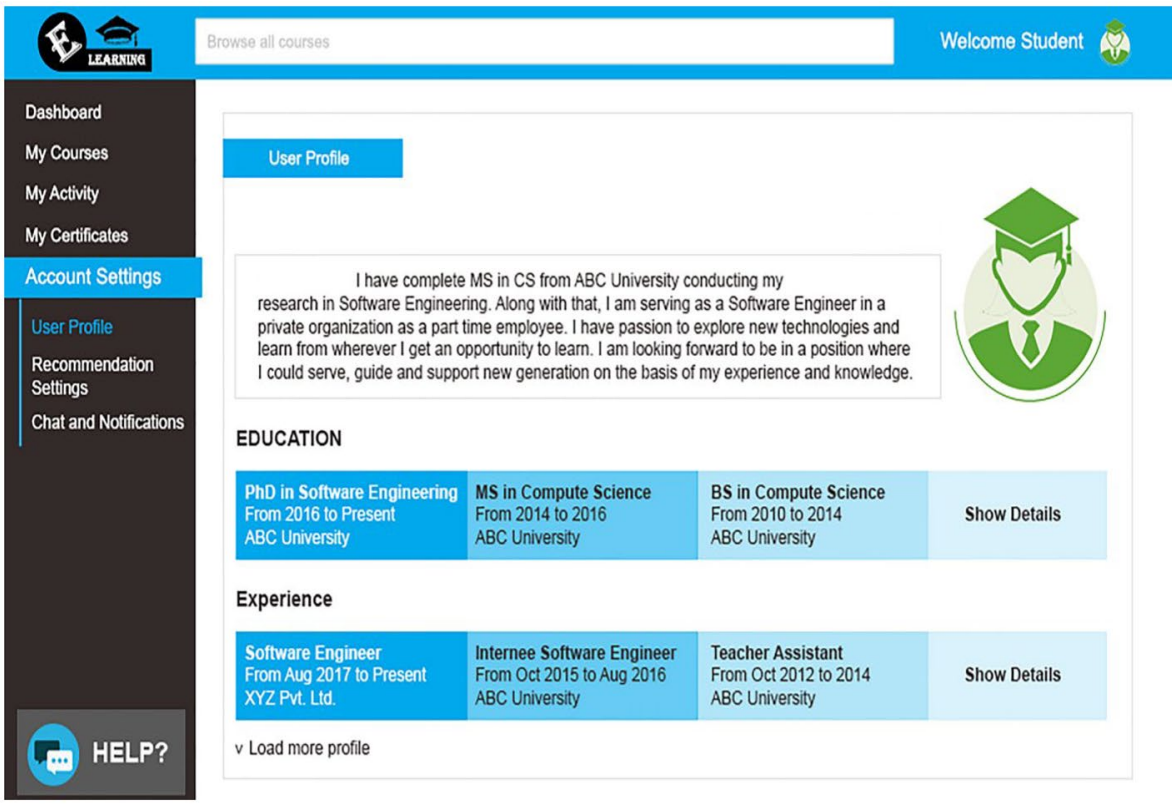

Fig. 2 Student Interface

\subsection{Input collection}

Data is collected from different users' profiles for their personalized preferences in the input collection phase. The student profile interface depicted in Fig. 2 shows the interface that defines background about education, skills, and experiences of an individual student. Based on student education and skills, the proposed system predicts the personalized preferences for online education. Personalize preferences are recommended using text mining semantic analysis. Later, utilizing similar prior and present teachers or students' ratings or feedback, a prediction list of solutions appropriate to preferences is presented.

\subsection{Prediction process}

In this phase, prediction and recommendation lists are generated virtually to enhance online education and identify the appropriate solution for users' online education problems. Virtual agent analyses prediction list based on previous and current similar users selected preferences for relevant issues solutions and feedback after adopting a solution. The previous and current user's similarity-based on teachers' or student's education, skills, and requirements. The highest-ranked solution predicts and recommends the new request of current or new users. The prediction list after the prediction process is depicted in Fig. 3. For an individual student, the personalized preferences prediction 


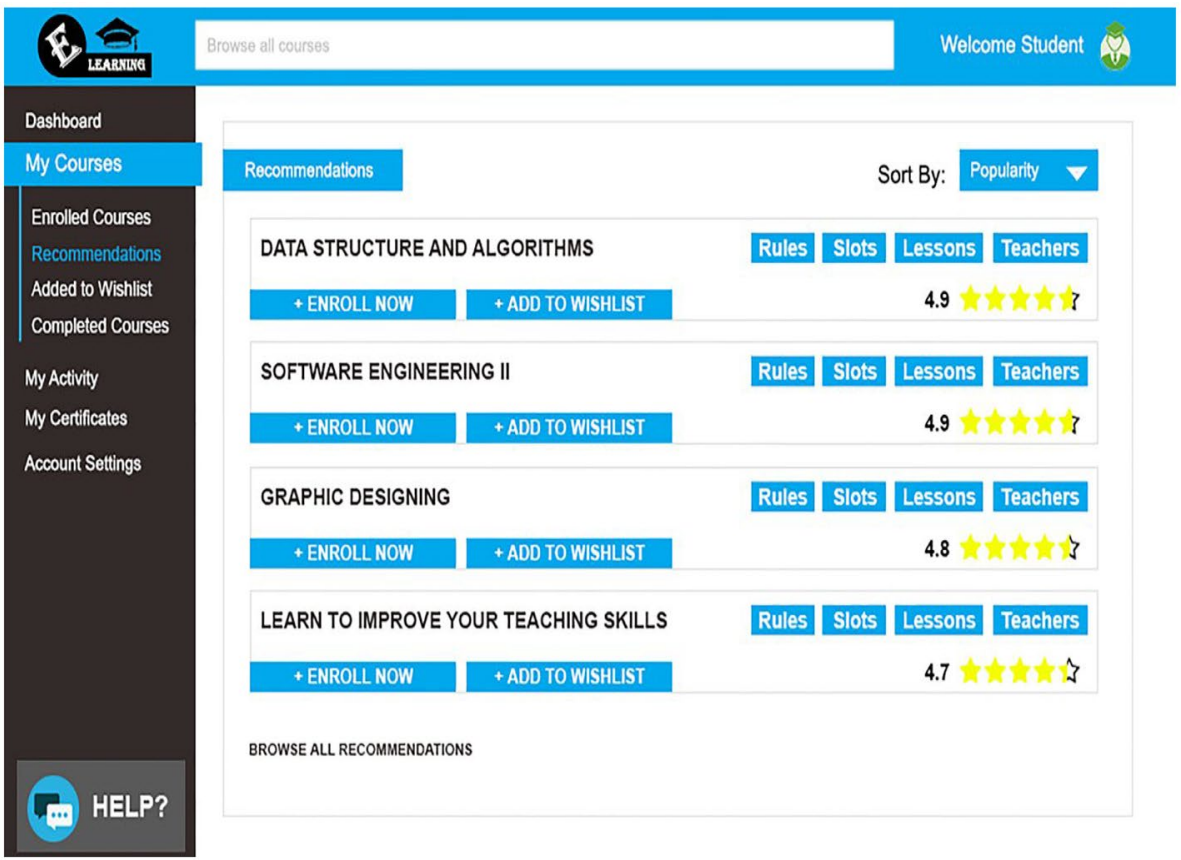

Fig. 3 Personalized Preferences Prediction Interface

interface presents the list of courses that improves knowledge by taking a course. For this phase, we use algorithm 2, and its input depends on algorithm 1.

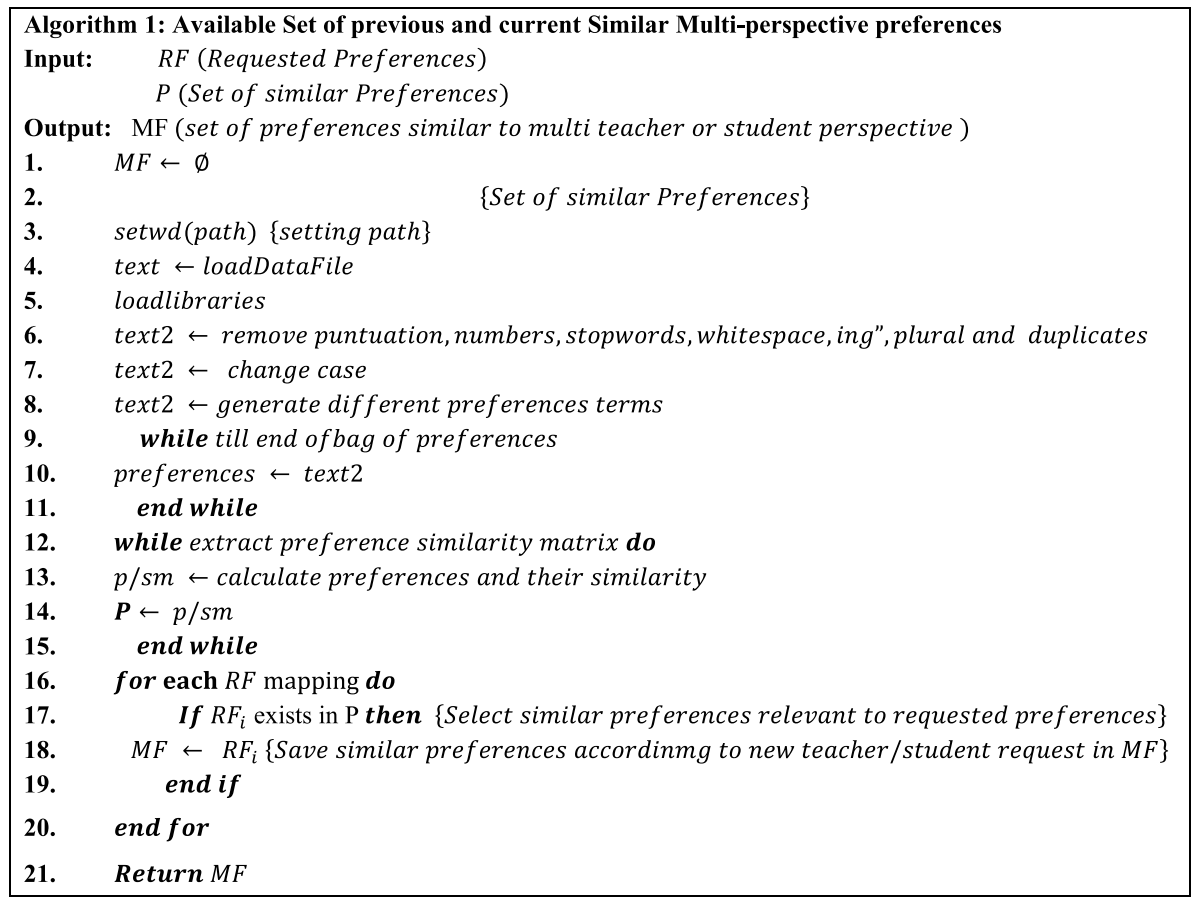




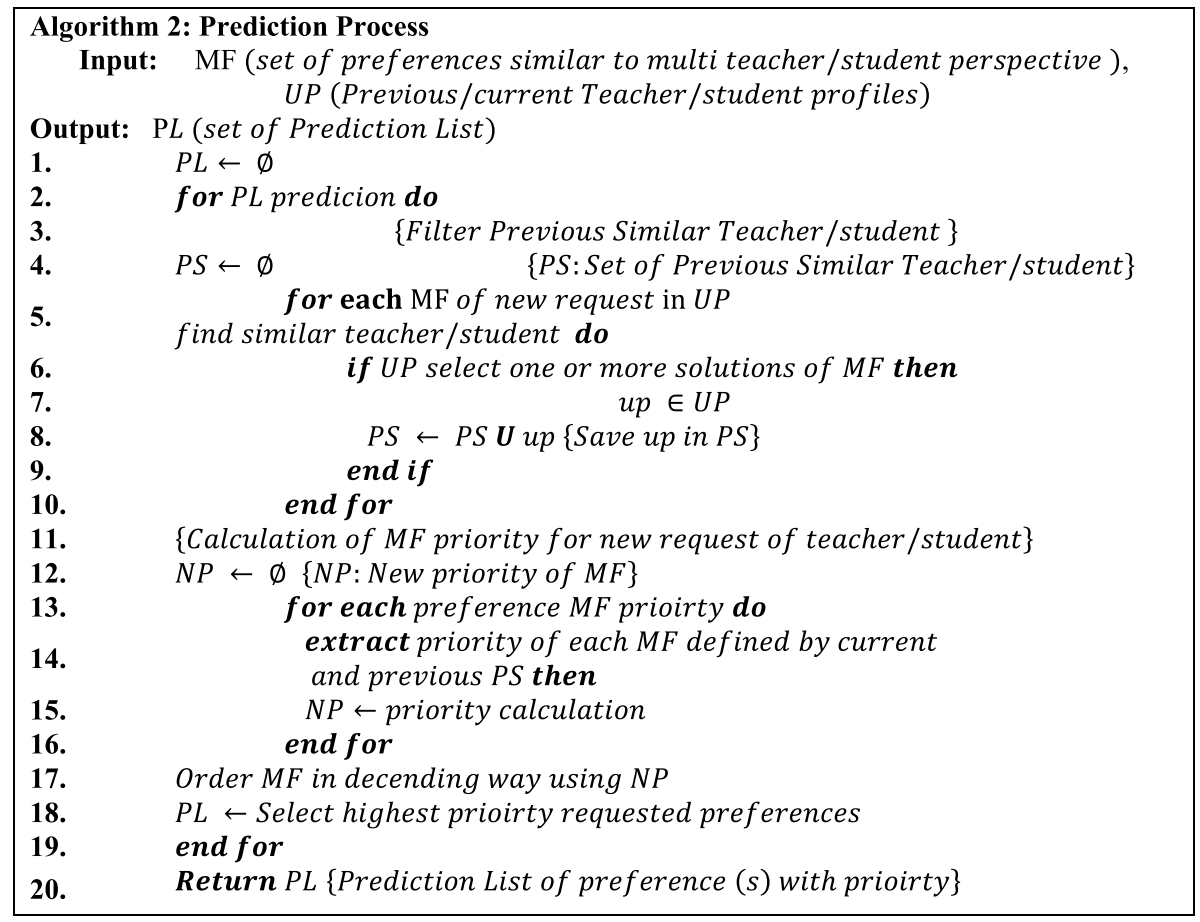

\subsubsection{Text mining}

In this phase, teacher's or student's requests are semantically preprocessed using text mining methods. For automatic text mining, we used R libraries using RStudio for semantic personalized preferences extraction $[11,29,32,41]$. Text mining is a type of data mining used to understand and find hidden relations in natural language text semantically [11, 29, $32,41]$. After the preprocessing, the data is stored in a repository. The full-text mining flow is illustrated in Fig. 4. Preprocessing phase is because the lack of information in user viewpoints is reduced, and the hidden relationship between the information provided can be

Fig. 4 Steps for Text Mining

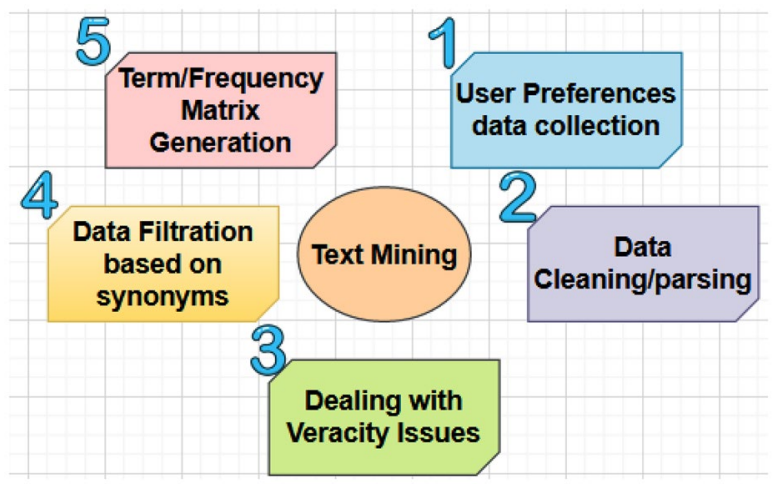




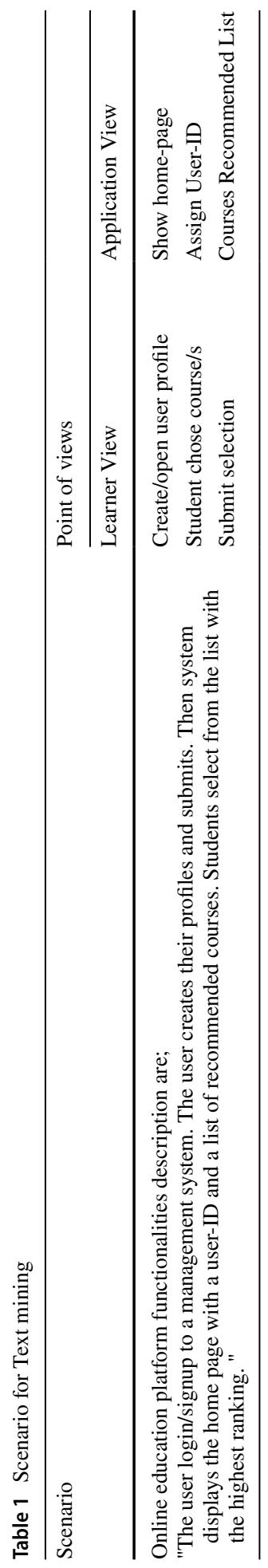


investigated or highlighted, or even recognized. Table 1 provides an example of the extraction of terms from the text of natural languages to concepts.

\subsubsection{Ranking preferences}

The ranking preferences are based on filtration of previous and current similarity of teachers and student's information. The filtration skills, experience, and qualification of new and current teachers or students are matched with the profile of the former similar students/teachers' experiences based on their semantic perspective analysis during online education. As most teachers/students are new and institutions to technology relevant to online education have shifted from campus education to online education overnight. Therefore, they needed guidance and training to select appropriate online mediums for education and virtual assistance for dealing with their issues based on personalized perspectives or preferences. The most adopted software for online education is Google classroom, Zoom, Microsoft team, Gmail, hangouts, and learning management systems. Most teachers and students are not familiar with these tools, and they face difficulties in adopting and maintaining their education standards. For example, most institutions adopted google classroom as an online medium for communication among teachers and students during online education. The teachers upload course contents, assignments, quizzes and handle queries using google classroom. And students have to follow teachers' instructions for content, assignments, etc. and communicate using this medium based on dispersed locations. Thus, teachers/students to enhance activities online education have to get training or resolve their problems while using these technologies.

Consequently, they used different social media and websites to enhance their skills but did not get accurate solutions according to their problems. For example, the quizzes conduction by teachers in google classroom is a problem, and after searching from different internet sources solution. The searching of solution wastage time and not accurately according to preferences. The proposed system extracts different solutions according to their personalized preferences about quiz conduction and submission issues. In the pandemic situation, they also utilize their time by taking short courses or training to enhance knowledge and skills. For example, the teacher/ student wants to enhance web development skills. Based on their previous qualification and experience, analyze and ranked according to current and previous similar users' feedbacks and preferences to predict different short courses using algorithms 1-1 to compete in their brighter career. Firstly, these courses extract by analyzing the request of teacher/student semantically in the form of terms or required preferences. Secondly, match current and previous similar students/teachers' personalized preferences according to their experiences and viewpoints to predict requirements. Thirdly, sort predicted options according to the ranking of previous and current teachers/ students.

\subsubsection{Prediction and priority}

After extracting preferences semantically and filtering according to similarity terms, predict list preference (s) with their priority in algorithm 2 . For the prediction list of preferences, extract previous teacher/student preferences and priority about similar preferences 
Table 2 Priority of Preferences

\begin{tabular}{llllll}
\hline Preferences & $P_{1}$ & $P_{2}$ & $P_{3}$ & $P_{4}$ & $P_{5}$ \\
\hline CP & 4 & 3 & 4 & 4 & 5 \\
PP & 2 & 3 & 1 & 3 & 1 \\
NP & 8 & 9 & 4 & 12 & 5 \\
\hline
\end{tabular}

$C P$ currently prioritized, $P P$ previously prioritized, $N P$ new priority and, $P$ Preferences

to classify preferences according to the highest priority. Therefore, the NP of predicted preferences with priority is a combination of PP as shown in Table 2 and $\mathrm{CP}$ preferences using Eq. 1.

$$
N P=\frac{\sum_{m=1}^{l} V_{m}}{z} \times C P
$$

whereas,

$V$ represents similar preferences set; $l$ is a preferences total number; $z$ characterizes total preferences selected by a user, $C P$ represents a number of users who recently have chosen a preference $V i$. According to Table 2, a new sequence of preferences are; $P_{4}, P_{2}, P_{1}, P_{5}, P_{a}$ using Eq. 1.

The proposed system assists virtually with the help of a virtual agent to resolve issues of teachers/students during online education and guiding teachers/students regarding enhancing their skills and experiences. To validate the performance of the proposed system; research questions used:

- Does the proposed system capable of improving remote education?

- Does the proposed system assist appropriately for the selection of a personalized environment during remote education?

\section{Experimentation and evaluation}

We conducted a quasi-experiment to evaluate the Proposed system (PS) using experimental group treatment (ET) and control group treatment (CT) by dividing participants in each group. The PS was implemented during the Covid-19 crisis in the lockdown situation to enhance users' skills to promote the idea of high-quality remote education to cope with the challenges of their access to quality education.

\subsection{Demographic information of participants (Ps)}

There were 60 participants, including students, virtual agents, teachers, and admin managers, for the conductance of online education during the experiment. These participants may or may not have online education experience before situating to the online 
Table 3 Demographic information

\begin{tabular}{llll}
\hline Experience & & CT Ps & ET Ps \\
\hline$<\&=2 \mathrm{Y}^{*}$ & Oc $* *$ & 10 & 9 \\
& Ol*** & 15 & 14 \\
$<\&=3 \mathrm{Y}$ & Oc & 6 & 7 \\
& Ol & 10 & 12 \\
$<\&=4 \mathrm{Y}$ & Oc & 5 & 6 \\
& Ol & 3 & 2 \\
$<\&=5 \mathrm{Y}$ & Oc & 6 & 6 \\
& Ol & 1 & 1 \\
$<\&=6 \mathrm{Y}$ & Oc & 3 & 2 \\
& Ol & 1 & 1
\end{tabular}

$\overline{\text { CT Ps Control Treatment preferences, ET Ps Experimental treatment }}$ preferences

*Y Years; **Oc On-Campus; ***Ol Online

education system, as described in Table 3. For experiments, selected institutions shifted from on-campus to online education after the impact of the current pandemic situation. The Ps of ET were 30 who implemented the proposed system (PS). At the same time, in CT, 30 participants included those who used the existing method (EM), i.e., existing websites like YouTube and Google without virtual assistance. The recruitment information participants are as follow:

- The participant's selection was based on random selection and consisted of both male and female individuals.

- The selected students are undergraduate students of the agriculture, mathematics, and computer science departments.

- The teachers of these departments were selected based on their experience in teaching.

- The virtual agents have experience of online education, modern technology, and prediction calculation semantically.

Therefore, all participants had different levels of experience, education, expertise, and skill. The selected participants from academia, including teachers and students, were connected with the educational institutes and their institutes adopted remote/online learning methods using Zoom, Google classroom, institution-based learning management systems, etc. The total period of the experiment, including from participants selection to output findings, consisted of 30 days. Course contents for short courses were selected from different websites and information about technology relevant preferences from institution repository they made before experiments after shifting to online education previously.

\subsection{Experimentation procedures}

In the spring session of the selected institution, the experiment was conducted for regular and short courses. The objective of PS is to predict a recommendation list of relevant resources to teachers and students according to their multiple perspectives. The prediction list with their 


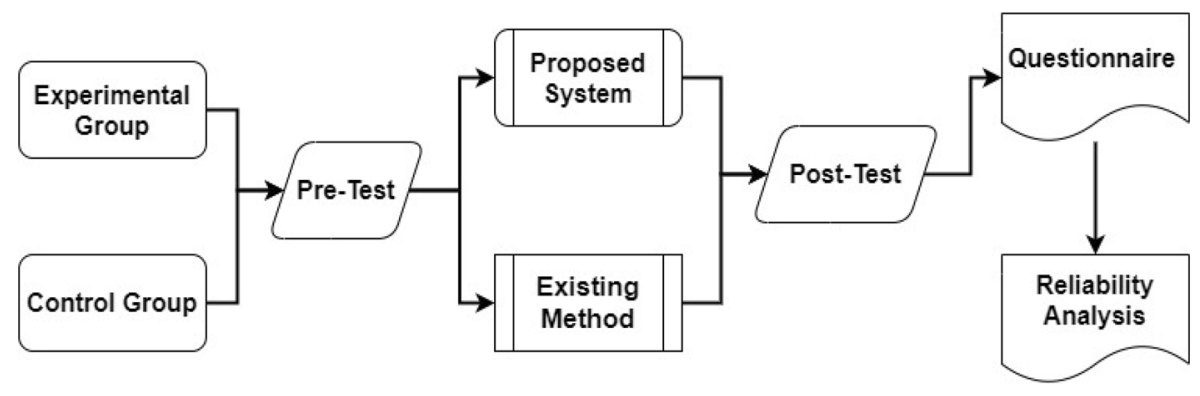

Fig. 5 Experiment Procedure

priority assists participants in enhancing their knowledge, experience, and skills during online education with virtual personalized preference assistance. For data collection questioner used as an instrument as described in Appendix A and the complete procedure of the experimental study described in Fig. 5. Therefore, after the division of participants, we applied pre-test, and after some essential training to get familiar with the proposed system, we applied post-test.

Questioner output generated from users' responses was then used to analyze statistically after the pre-post-tests. The measurement of the experiment is based on participants' satisfaction and novelty effectiveness of the proposed system. Thus, we used parametric evaluation for the satisfaction of participants, which was extracted from existing literature, i.e. [10, 12, 14, 16, 23, 30, 30, 31]. These parameters are described in Table 4, and data collection about these parameters from participants' feedback and experience based on the questioner.

The pre-post-tests were created to look into the effectiveness of the suggested system during online education and to assess teachers and students' reactions after using it. Therefore, after the division of participants, we assess the previous knowledge, skills, and familiarity with technology and reviews about online shifting overnight of participants using questioner. The content of pre and post-test were in the form of videos and slides which were used by the participants for their issues during the use of remote education or studying short courses for skills and knowledge enhancement. These contents were saved in the repository,

Table 4 List of Factors

\begin{tabular}{ll}
\hline S. \# & Factors \\
\hline i & Easily Understandable (EU) \\
ii & Less Complicated (LC) \\
iii & Student/learner Performance level (SP) \\
iv & Increase/Boost Motivation (IM) \\
v & Reduce team Efforts (RE) \\
vi & Learning ability (Le) \\
vii & Cooperation online (Co) \\
viii & Social relations Enhancement (SE) \\
ix & Personalized selection (Pe) \\
X & User Satisfaction (US) \\
xi & Semantically analysed Info (SI) \\
xii & Virtually aided Environment (VE) \\
xiii & Use Valuable Preferences (UP) \\
xiv & Accurate and correct Referred preferences (AR)
\end{tabular}


Table 5 Statistical test

\begin{tabular}{llllllr}
\hline Groups & Ps & \multicolumn{2}{l}{ Pre-Test } & & \multicolumn{2}{l}{ Post-Test } \\
& & U & S.D & & U & \multicolumn{2}{l}{ S.D } \\
\hline Control Treatment & 30 & 28.1 & 9.18 & & 36.5 & 9.55 \\
Experimental Treatment & 30 & 37.6 & 10.1 & & 60.1 & 12.05 \\
\hline
\end{tabular}

Ps Preferences, $U$ Mean, SD Standard Deviation

and the virtual agent helped to access these contents after the selection of desire contents. The pre-test and training of participants consist of 5 days, 15 days for learning of contents, and ten days for post-test data collection, results in analysis, comparison with pre-test, and conclusion. This assessment helps to compare results after implementing treatment and improvement achieved. The CT and ET participants have different online and on-campus experiences with less satisfaction with shifting to online education. Most participants who are not familiar with advanced technology do not have access to all online resources, face difficulties while communicating with students and teachers due to the involvement of multiple tools. The results of CT and ET participants almost the same because most of the participants have no or less experience with online education software. During the experiment, CT group enhanced their skills using different tutorials and materials using different online platforms, including YouTube and Google. While all participants of ET group adopted PS to enhance their skills, resolve the challenges of Covid-19 on education and complete their educational requirements without affecting their lives in pandemic impact.

After applying treatment to ET participants, a post-test was performed on responses. Therefore, before applying a treatment of proposed system treatment, provide training to ET about using the proposed system and enhance knowledge of CT participants for correct and accurate assessment of post-test. The results of the pre-post-test were then compared to identify the differences between the pre-post-test values for validation after treatment.

\subsection{Results and discussion}

This part of study explains experiment conduction procedure to assess performance of CT and ET participant's, novel effects, and standard education after implementing PS. For analyzing data, collected after pre and post-test, using SPSS software version 23 as shown in Table 5 and for reliability analysis of data used Cronbach's alpha test as shown in Table 6 to compare significant difference and reliability analysis results of pre and post-tests.

CT and ET mean 28.008 and 37.6, respectively, with a standard deviation (S.D.) of 9.18 and 10.1. The reliability test is acceptable when the post-test value is greater than the pre-test alpha value. Thus, results for the pre-test of CT and Et are 0.69 and 0.76, respectively. Subsequently, the post-test of CT and ET are 0.78 and 0.89 , respectively. In order to analyze the S.D between CT and ET, an independent or paired t-test was carried out

Table 6 Cronbach's statistical alpha test

\begin{tabular}{lllllll}
\hline Groups & Preferences & Pre-Test & & \multicolumn{3}{l}{ Post-Test } \\
\hline & & t-value & & Significance-value & t-value & significance-value \\
Control Treatment & 30 & 2.75 & 0.001 & & 2.989 & 0.000 \\
Experimental Treatment & 30 & 3.047 & 0.000 & 3.867 & 0.000 \\
\hline
\end{tabular}


Table 7 Statistic Analysis

\begin{tabular}{llllll}
\hline G & SS & $\begin{array}{l}\text { Degree of } \\
\text { freedom }\end{array}$ & Mean Square & F & $\begin{array}{l}\text { Significance } \\
\text { difference }\end{array}$ \\
\hline $\begin{array}{l}\text { Between Control and } \\
\text { Experiment Treatments }\end{array}$ & 1978.733 & 14 & 141.338 & 3.750 & 0.001 \\
$\begin{array}{l}\text { Within Control and } \\
\text { Experiments Treatments }\end{array}$ & 2018.467 & 15 & 134.564 & \\
\begin{tabular}{l} 
Total Value \\
\hline
\end{tabular} & 3997.200 & 29 & & \\
\hline
\end{tabular}

$G$ Groups, SS MeanSum of squares

to evaluate the hypotheses $(\mathrm{H})$ and the significant difference between the group pre-posttest. The significance-value (sv), i.e., $<0.05$, shows that Ps satisfaction and performance increase after the proposed system has been implemented. As an outcome, reject $\mathrm{H}$ null, indicating that participants in ET and CT had diverse levels of education, experiences, talents, and so on $(t=3.867, s v=0.000)$. Consequently, it also concludes that semantic-based predictions in virtualized environments improved the selection of relevant, personalized preferences and significantly outperformed the existing method.

We performed a one-way ANOVA test to compare dependent (performance, satisfaction level, and novelty) and independent factors (i.e., semantic-based personalized preferences) as described in Table 7 between ET and CT. The results demonstrate that sv difference between PS and EM $(F=3.750, \mathrm{sv}=0.001)$, with mean square values 141.338 and 134.564, respectively. On the other hand, ET mean score was greater than CT, demonstrating that it is possible to improve the online course selection process by considering many perspectives. The ET performed better in terms of score, as evidenced by descriptive analysis.

In addition to the results of a questionnaire prove that PS outperform than EM, as shown in Fig. 6 and 7 for CT and ET, the participants' satisfaction level (SL) outcomes. Ps' level of satisfaction is represented on the x-axis, while the number of Ps is represented on the y-axis. The value of satisfaction level of EM or CT group data calculated using questioner instrument to compare results of EM and PS participants. Therefore, Fig. 6. depicted the EM participants' scores which calculated using an average of questioner data based on five Likert scales for Ps SL after the experiment. Thus, SL of an instructor, student, and manager less than 65 percent as compared to PS participants, which is greater than 65 percent (see Fig. 7).

Fig. 6 EM Participants Results

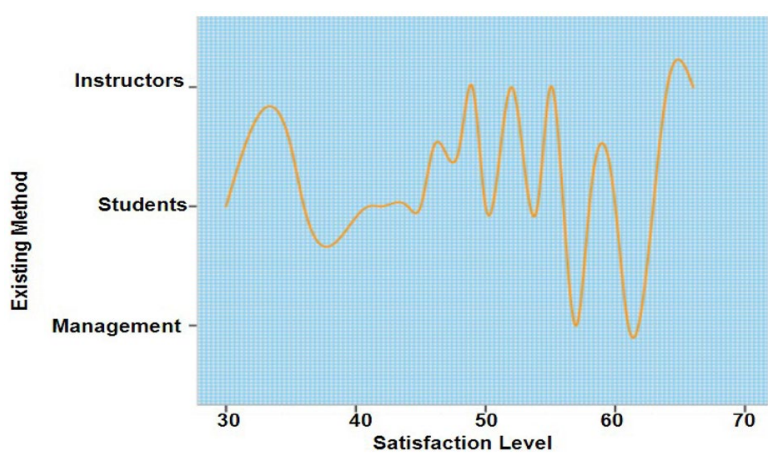


Fig. 7 PS Participants Satisfaction Level

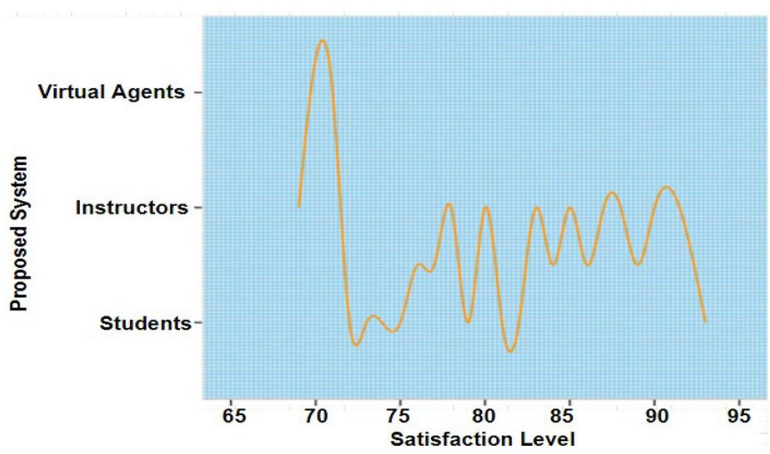

PS updated the online education platform and provided personalized semanticbased predictions on diverse perspectives, according to the respondents' results. The proposed system, alternatively, lowered the effort needed for recommending highquality courses. In addition to this, participants were provided an interactive virtualized learning environment/platform as well. If a student has a question during class, quizzes, or he/she want to ask anything during the exam, for example, virtual support will address problems and assist them. It enables remote learning more engaging for short-term courses after mapping the recommendation list according to their interests/ wishes. The ET teachers were also able to complete and provide high-quality education within the required time and were able to solve the queries and problems of their students. Similarly, ET students never lose their interest, and they keep on their participation efficiently during remote learning in a virtual-aided scenario under the guidance of their teachers. Thus, it kept on providing high-quality education without losing the interest of instructors and students remotely during the pandemic. According to each Likert scale, the PS and EM findings were then provided in Figs. 8, 9, 10, 11, 12 as indicated in Appendix A.

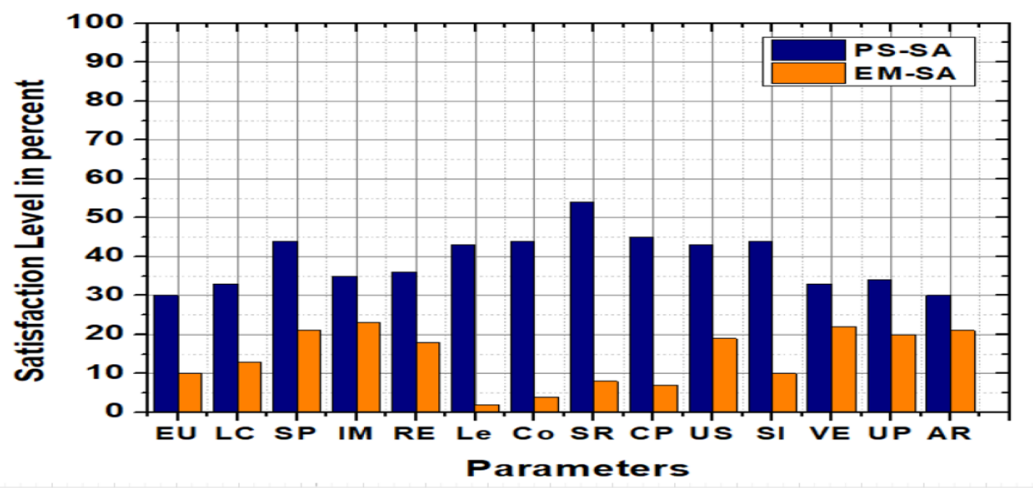

Proposed System (PS), Existing Method (EM), Strongly Agreed (SA),

Easily Understandable (EU), Less Complicated (LC), Student/learner Performance level (SP), Increase/Boost Motivation (IM), Reduce team Efforts (RE), Learning ability (Le), Cooperation online (Co), Social relations Enhancement (SE), Personalized selection (Pe), User Satisfaction (US), Semantically analyzed Info (SI), Virtually aided Environment (VE), Use Valuable Preferences (UP), Accurate and correct Referred preferences (AR)

Fig. 8 Parameters Analysis of Strongly Agreed 


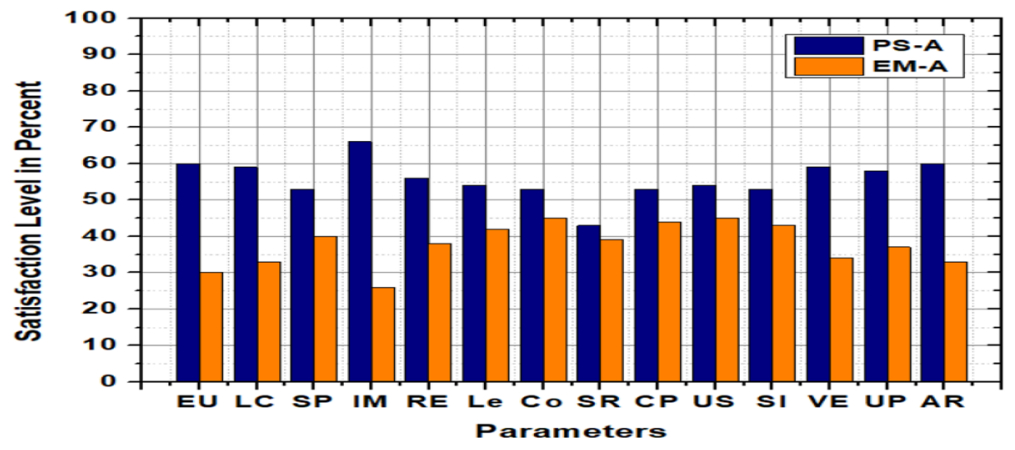

Proposed System (PS), Existing Method (EM), Agreed (A),

Easily Understandable (EU), Less Complicated (LC), Student/learner Performance level (SP), Increase/Boost Motivation (IM), Reduce team Efforts (RE), Learning ability (Le), Cooperation online (Co), Social relations Enhancement (SE), Personalized selection (Pe), User Satisfaction (US), Semantically analyzed Info (SI), Virtually aided Environment (VE), Use Valuable Preferences (UP), Accurate and correct Referred preferences (AR)

Fig. 9 Parameters Analysis of Agreed

As a result, the average satisfaction levels of all participants found in Figs. 8, 9, 10, 11 and 12 for each parametric expression used $\mathrm{n}$ in Table 4 for comparison. This revealed that all PS participants were happier than those who used Ps, which is based on a typical online learning technique. As a result, PS outperforms EM and enhances educational performance to the best possible level.

\subsection{Novelty effect}

The ability to detect human learning ability with the help of new technology and familiarity with technology contents to memorize easily and better as compared to other methods

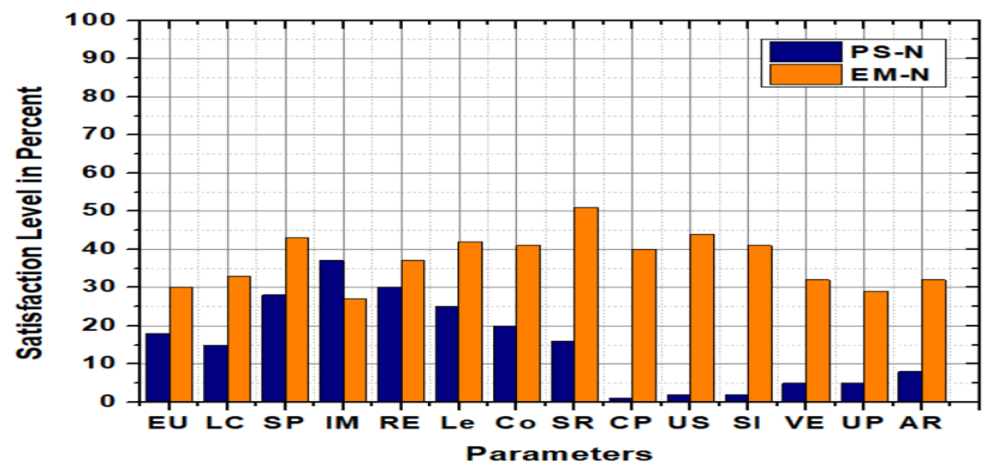

Proposed System (PS), Existing Method (EM), Neutral (N),

Easily Understandable (EU), Less Complicated (LC), Student/learner Performance level (SP), Increase/Boost Motivation (IM), Reduce team Efforts (RE), Learning ability (Le), Cooperation online (Co), Social relations Enhancement (SE), Personalized selection (Pe), User Satisfaction (US), Semantically analyzed Info (SI), Virtually aided Environment (VE), Use Valuable Preferences (UP), Accurate and correct Referred preferences (AR)

Fig. 10 Parameters Analysis of Neutral 


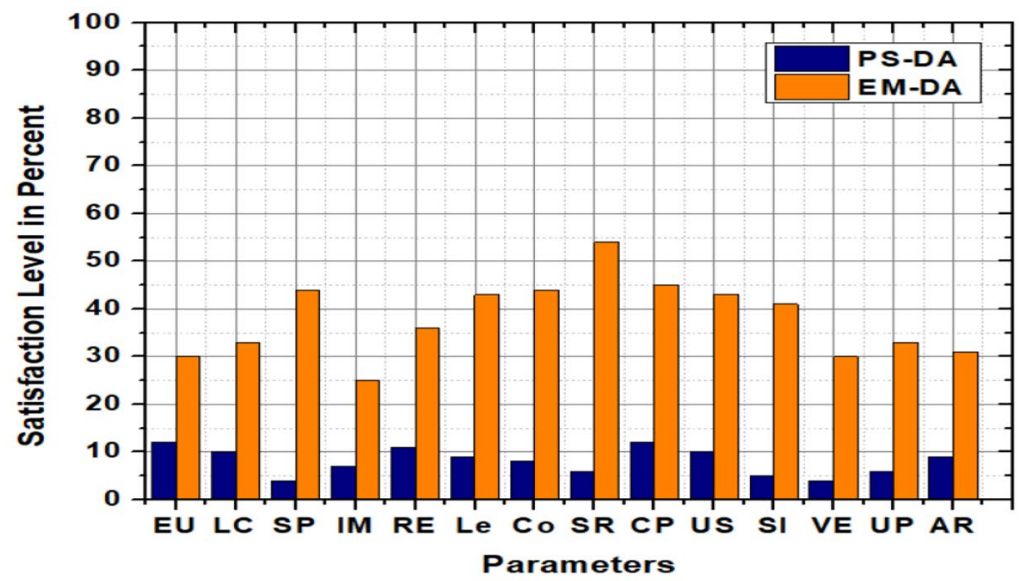

Proposed System (PS), Existing Method (EM), Disagreed (DA),

Easily Understandable (EU), Less Complicated (LC), Student/learner Performance level (SP), Increase/Boost Motivation (IM), Reduce team Efforts (RE), Learning ability (Le), Cooperation online (Co), Social relations Enhancement (SE), Personalized selection (Pe), User Satisfaction (US), Semantically analyzed Info (SI), Virtually aided Environment (VE), Use Valuable Preferences (UP), Accurate and correct Referred preferences (AR)

Fig. 11 Parameters Analysis of Disagreed

$[21,28,33,42]$ has also been tested in this work. In this research, we evaluate the novelty effect of PS as compared to EM in case of performance, personalized selection, content, flexibility, innovation, interest, and learnability according to Ps experiences using data collected after the questionnaire. The results depicted in Fig. 13 show that using PS participant's motivation, knowledge, interest, learning ability, and the ratio of successful achievement of their goals has been improved as compared to EM.

\subsection{F measure and accuracy}

In order to evaluate the success of PS to identify appropriate, relevant predictions and selection of personalized preferences used F-measure and accuracy metrics [24, 40]. Accuracy measures exactness in prediction priority personalized preferences using semantic analysis. The F-measure used to quantity relationship between predicted preferences and actual relevant predictions. Subsequently, results show that PS and EM accuracy are 0.56 and 0.98 , respectively, and PS accuracy and F-measure are 0.59 and 0.98 values, respectively. Thus, the predictions of personalized preferences are correctly prescribed to teachers/students according to their requirement and perspective semantically than the existing method.

After the experiment, the overall satisfaction level of participants has increased, as depicted in Fig. 14. The axis $y$ and $x$ describe the SL and participants' detail to evaluate the performance of each participants. And results depicted that PS participants have more than 70 percent SL as compared to EM.

We investigated whether preference prediction in an e-learning environment benefited the online learning process by selecting relevant preferences based on user interest and 


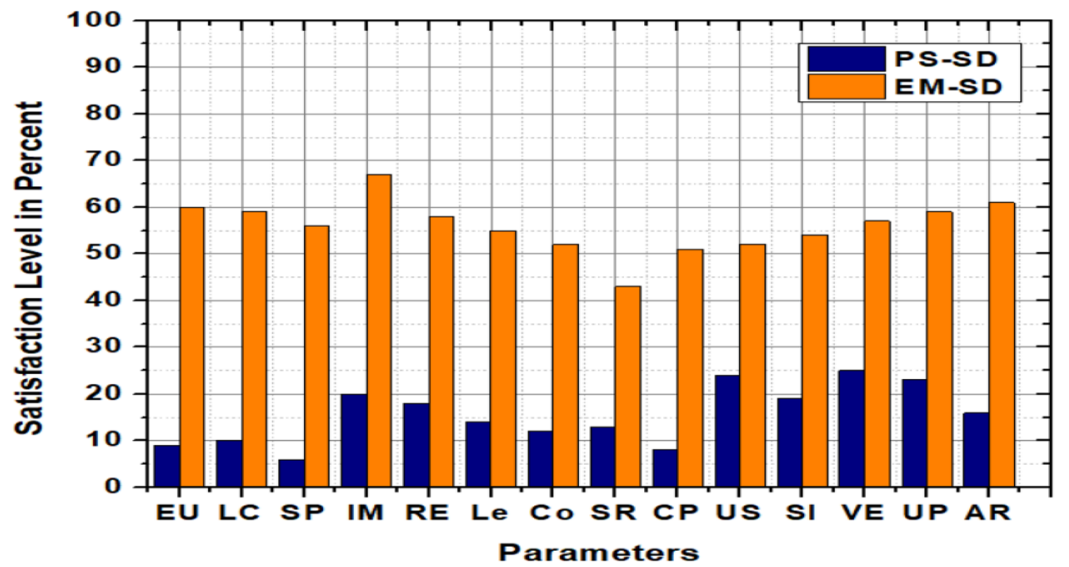

Proposed System (PS), Existing Method (EM), Strongly Disagreed (SD),

Easily Understandable (EU), Less Complicated (LC), Student/learner Performance level (SP), Increase/Boost Motivation (IM), Reduce team Efforts (RE), Learning ability (Le), Cooperation online (Co), Social relations Enhancement (SE), Personalized selection (Pe), User Satisfaction (US), Semantically analyzed Info (SI), Virtually aided Environment (VE), Use Valuable Preferences (UP), Accurate and correct Referred preferences (AR)

Fig. 12 Parameters Analysis of Strongly Disagreed

preferences in the current situation when students and teachers have migrated to online education overnight. As a result, the statistical test reveals a significant difference between the proposed system and the present approach in order to answer the study question: "Does the suggested system increase e-learning?" In the advance technical era, the selection of semantically diverse perspective preferences in VE is important to increase motivation and capabilities. Existing information-search systems frequently offer the same set of

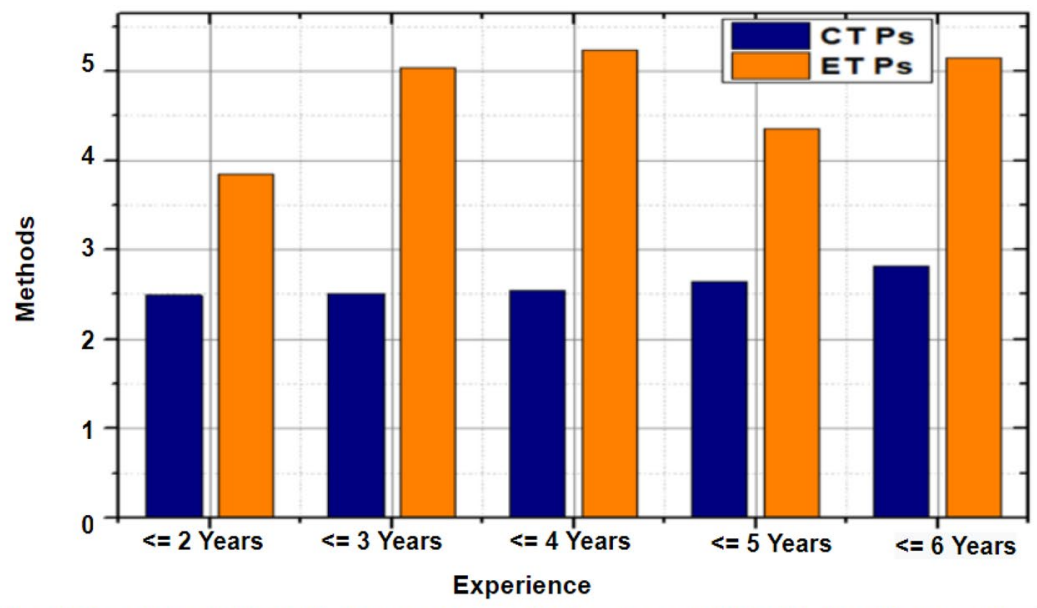

Control Treatment (CT), Experiment Treatment (ET), Participants (PS)

Fig. 13 Novelty effect 


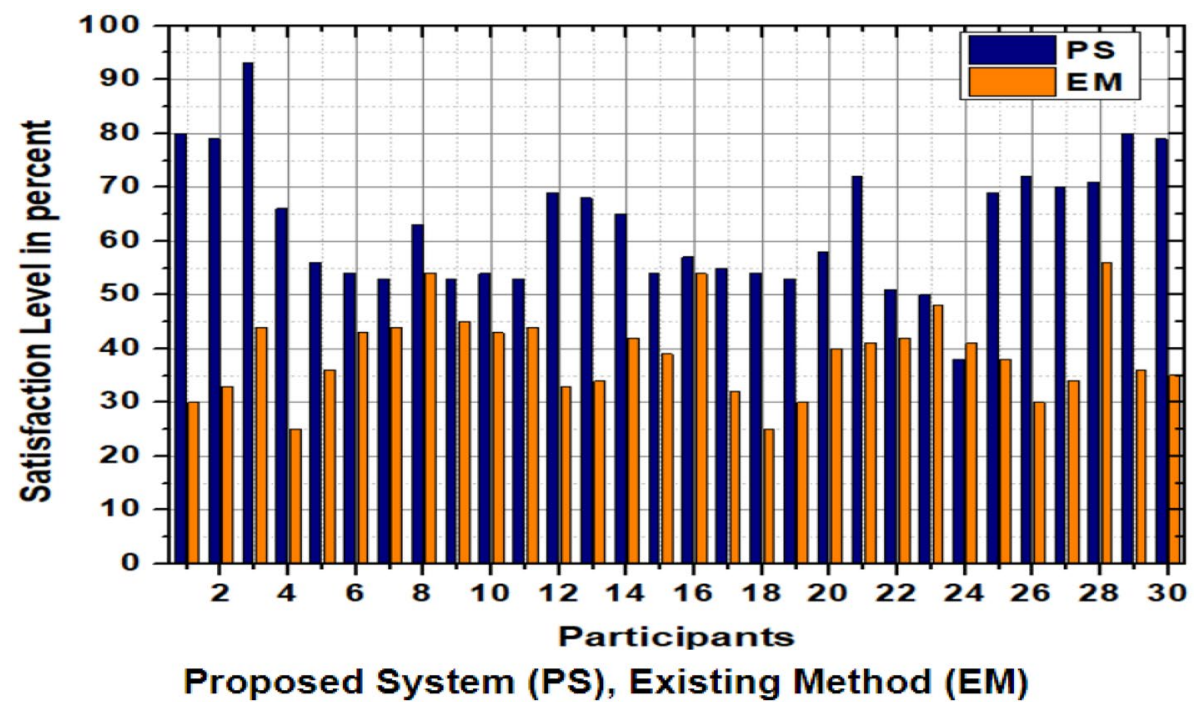

Fig. 14 Comparison of PS and EM

information, as well as irrelevant and redundant information, to prospective user preferences all at once, without virtual help, obstructing their tailored preferences process. The implementation of a semantic-based and virtual assistance-based recommender system increases user motivation while also assisting in the development of abilities to meet institutional needs. Learners and tutors alike considered it enticing for learning and building their specialized/educational aids in a simulated dynamic environment to eagerly discover attractive and relevant courses. Furthermore, learners and instructors felt more confident and competent as a result of the ability to learn and teach syntax courses based on user and market demands.

Throughout the experimentation, it was noted that the CT group learners were uninterested/tired after choosing unrelated/immaterial courses from the existing choices, consequential decrease in learner/instructor performance in comparison to ET performance/ efficiency. Their slot may have been assigned to other students during the study, or the interface may have been down at the quiz time, and there was no online agent to help CT learners. While the identical problem that occurred with ET participants while using PS during learning was later resolved by virtual representative guidance, and there is less percentage of incorrect course choice. Henceforward, all ET participants had higher SL, irrespective of students, instructors, or virtual representatives, than CT group participants. As portrayed in the factors analysis, the PS users have an SL greater than 50 out of a hundred compared to conventional method participants whose SL was smaller than 50 out of a hundred.

Participants in the CT and ET groups reported varying SL while searching required material or tutorials using existing platforms (like Google and YouTube) and PS, respectively. The participants must choose the appropriate courses/materials using a single PS platform in accordance with their interest from the recommended list without wasting time searching relevant material from different sources/platforms to complete 
their educational target on time. Some instructors and/or students may lose their interest because after using a different medium, they may fail to find the required material available due to a lack of virtual guidance. In contrast, ET participants used PS platform to mitigate issues related to remote learning and increased learners' interest to get solutions of their queries on time whenever required by ET participants. Because existing methods/platforms never provide virtual help and diverse perspective-based materials. And PS recommends a list of material according to previous participants' experience with the help of a virtual guided environment. As a result, PS improves their skills and motivates ET participants to provide and get high-standard education in the global crisis where all the physical activities were completely suspended. The reasons to experiment for a short period instead of a longer period are; to facilitate institutions to run their education system smoothly and to cope with the hurdles of a sudden change in the working environment due to Covid-19 and provide assistance to participants to handle remote education challenges.

\section{Conclusion and future work}

The research work is a contextual analysis and virtually aided agent-based system that helps students/tutors to find and select relevant learning/teaching training programs to enhance participant's skills grounded on their benefits and preferences in the lockdown situation during Covid-19. As in Covid-19, economic and academic challenges have been increased due to the shortage of resources because of the suspension of all kinds of export, import, and social contacts with others worldwide. Due to this crisis, it is difficult to cope with the highlighted issues globally, and the education sector, in particular, has been affected in developing countries. Thus, institutions have to adopt innovative ideas and procedures for remote learning, and different available tools like Zoom, MS team, Hangouts, etc., could be used for this purpose. The main issue with the adoption of these measures during remote learning was that most of the tutors and students have no experience and familiarity with remote learning. Therefore, the proposed system helped these tutors/students for enhancing their skills and knowledge to provide high-quality education. Thus, the proposed system takes a number of perspectives into account when selecting relevant and inclusive learning content to enhance learners' abilities, knowledge, and online learning methods.

Moreover, two important impacts of the proposed system are: helping students and teachers to select suitable advanced courses that develop their knowledge and knowledge in line with their interests and needs. Second, the suggested system addresses online user queries to eliminate the gaps in user's coordination and ambiguity, resulting in high-quality teaching. Validation of proposed methods and their comparison with other techniques shows that the proposed system strategies have greatly improved the abilities and achievements while also greatly improving learning performance (by more than 90\%) as compared to other traditional ways. Our future research will focus on improving the suggested system for dynamically updating syllabus and learning material and validating student and teacher performance to increase the quality of online education. The proposed system also improved the assessment of students' knowledge 
using different artificial intelligence methods to discourage plagiarism-based activates during the online examinations.

\section{Appendix A: Questioner}

The appendix provides information about questions used for data collection during experimental study. Thus, questioner divided into three sections.

\begin{tabular}{|c|c|c|c|c|c|}
\hline \multicolumn{6}{|c|}{ Section 1: Demographic Information of Participants } \\
\hline \multicolumn{6}{|l|}{ Name: } \\
\hline \multicolumn{6}{|c|}{ Qualification: } \\
\hline Teacher: & Student: & \multicolumn{2}{|c|}{ Virtual Agent: } & Manager: & \\
\hline Gender: & \multicolumn{2}{|l|}{ Male } & \multicolumn{2}{|l|}{ Female } & \\
\hline Age: & $<\&=18$ Years & $<\&=25$ Years & $<\&=35$ Years & $<\&=45$ Years & $<\&=60$ Years \\
\hline Experience: & $<\&=2$ Years & $<\&=3$ Years & $<\&=4$ Years & $<\&=5$ Years & $<\&=6$ Years \\
\hline \multicolumn{6}{|l|}{ On Campus: } \\
\hline Online: & & & & & \\
\hline
\end{tabular}

Then participants fill Sect. 2 to provide review about proposed system performance and satisfaction level. Hence Sect. 3 used to identify proposed system novelty effect. For Sects. 2 and 3 used five liker scales used selected i.e. 1=Strongly-Agreed (SA), $2=$ Agreed (A), $3=$ Neutral (N), $4=$ Dis-agreed (DA), and $1=$ Strongly-Disagreed (SD). The participants ranked them from 1 to 5 points ranking. 
Section 2: Performance and Satisfaction Level

\begin{tabular}{llll}
\hline No. Questions & Scaling & \\
\cline { 2 - 3 } & SA A N DA SD \\
\hline
\end{tabular}

Q1 Does proposed system provide easy to understand and manage education standard?

Q2 Does proposed system is suitable and less complex to improve online education?

Q3 Does proposed system improve student performance during online education period?

Q4 Does proposed system increase motivation of teacher/students for online education?

Q5 Does proposed system reduce effort during online education?

Q6 Does proposed system provide a personalization mechanism to support teachers/students in order to facilitate interactive online education current pandemic situation?

Q7 Does proposed system have capability to enhancing coordination among teachers and students using virtualized environment?

Q8 Does the social enhancement increase using proposed system in order to formulate new method?

Q9 Does proposed system increase learnability by providing personalized preferences based on multi perspective ultimately enhance overall standard and quality of education?

Q10 Does user satisfaction facilitate teachers and students to enhance their skills, experience and knowledge?

Q11 Does proposed system extract efficiently Semantic Information for accurate any complete personalized preferences?

Q12 Does proposed system is useful and to implement and trained teachers an education in virtualized environment?

Q13 Does the preferences predict by proposed system useful preferences during online education?

Q14 Does proposed system identify relevant and accurate recommendations for higher performance? 
Section 3: Novelty Effect

\begin{tabular}{llll}
\hline No. Questions & \multicolumn{2}{l}{ Scaling } & \\
& & \\
& SA A N DA SD
\end{tabular}

Q1 Does proposed system methodology new experience for you?

Q2 Does proposed system capable for identifying relevant and unique solution for personalized preferences in modern way?

Q3 Does you get accurate preferences prediction list semantically using proposed approach?

Q4 Does proposed system easy to use and implements desire preferences?

Q5 Does proposed system provide new way after text mining and participants previous behavior?

Q6 Does proposed system able to reduce gaps among theory and practices to implement in real scenario?

Q7 Does proposed system capable to manage and increase information semantically as compared to existing methods?

\section{References}

1. Aher SB, Lobo LMRJ (2013) Combination of machine learning algorithms for recommendation of courses in E-Learning System based on historical data. Knowl-Based Syst 51:1-14. https://doi.org/10. 1016/j.knosys.2013.04.015

2. Arias M, Buccella A, Cechich A (2018) A Framework for Managing Requirements of Software Product Lines. Electronic Notes in Theoretical Computer Science 339:5-20. https://doi.org/10.1016/j.entcs. 2018.06.002

3. Baloian N, Zurita G (2016) Achieving better usability of software supporting learning activities of large groups. Inf Syst Front 18:125-144. https://doi.org/10.1007/s10796-015-9580-3

4. Bank W (2020) Remote Learning, Distance Education and Online Learning During the COVID19 Pandemic. World 'Bank's Edtech Team. World Bank, Washington, DC. (C World Bank

5. Beer UM, Neerincx MA, Morina N, Brinkman W-P (2017) Virtual agent-mediated appraisal training: a single case series among Dutch firefighters. Eur J Psychotraumatol 8:1378053. https://doi.org/10. 1080/20008198.2017.1378053

6. Brigui-Chtioui I, Caillou P, Negre E (2017) Intelligent Digital Learning: Agent-Based Recommender System. In: Proceedings of the 9th International Conference on Machine Learning and Computing ICMLC 2017. ACM Press, Singapore, Singapore. 71-76

7. Campos R, Pereira dos Santos R, Oliveira J (2018) Web-Based Recommendation System Architecture for Knowledge Reuse in MOOCs Ecosystems. In: 2018 IEEE Interna Conf Inform Reuse Integ (IRI). IEEE, Salt Lake City, UT. 193-200

8. Cao W, Fang Z, Hou G et al (2020) The psychological impact of the COVID-19 epidemic on college students in China. Psychiatry Res 287:112934. https://doi.org/10.1016/j.psychres.2020.112934 
9. Cascella M, Rajnik M, Cuomo A et al (2020) Features, evaluation and treatment coronavirus (COVID19). In Stat Pearls Publishing

10. Cerezo R, Bogarín A, Esteban M, Romero C (2019) Process mining for self-regulated learning assessment in e-learning. J Comput High Educ. https://doi.org/10.1007/s12528-019-09225-y

11. Chabrun F, Huetz N, Dieu X et al (2020) Data-Mining Approach on Transcriptomics and Methylomics Placental Analysis Highlights Genes in Fetal Growth Restriction. Front Genet 10:1292. https://doi.org/ 10.3389/fgene.2019.01292

12. Chavarriaga O, Florian-Gaviria B, Solarte O (2014) A Recommender System for Students Based on Social Knowledge and Assessment Data of Competences. In: Rensing C, de Freitas S, Ley T, MuñozMerino PJ (eds) Open Learning and Teaching in Educational Communities. Springer International Publishing, Cham, pp 56-69

13. Choi C-R, Jeong H-Y (2019) Quality evaluation for multimedia contents of e-learning systems using the ANP approach on high speed network. Multimed Tools Appl 78:28853-28875. https://doi.org/10. 1007/s11042-019-7351-8

14. Dahdouh K, Dakkak A, Oughdir L, Ibriz A (2019) Large-scale e-learning recommender system based on Spark and Hadoop. J Big Data 6:2. https://doi.org/10.1186/s40537-019-0169-4

15. Elazony M, Khalifa A, Nouh S, Hussein M (2018) Design and Implementation of Adaptive Recommendation System. 3:17

16. Fraihat S, Shambour Q (2015) A Framework of Semantic Recommender System for e-Learning. JSW 10:317-330. https://doi.org/10.17706/jsw.10.3.317-330

17. Fridin M, Belokopytov M (2014) Embodied Robot versus Virtual Agent: Involvement of Preschool Children in Motor Task Performance. Interna J Human-Comp Interact 30:459-469. https://doi.org/10. 1080/10447318.2014.888500

18. Graics B, Molnár V, Vörös A et al (2020) Mixed-semantics composition of statecharts for the componentbased design of reactive systems. Softw Syst Model. https://doi.org/10.1007/s10270-020-00806-5

19. Hwang G-J, Chang H-F (2011) A formative assessment-based mobile learning approach to improving the learning attitudes and achievements of students. Comput Educ 56:1023-1031. https://doi.org/10. 1016/j.compedu.2010.12.002

20. Hwang G-J, Fu Q-K (2019) Trends in the research design and application of mobile language learning: a review of 2007-2016 publications in selected SSCI journals. Interact Learn Environ 27:567-581. https://doi.org/10.1080/10494820.2018.1486861

21. Jeno LM, Vandvik V, Eliassen S, Grytnes J-A (2019) Testing the novelty effect of an m-learning tool on internalization and achievement: A Self-Determination Theory approach. Comput Educ 128:398413. https://doi.org/10.1016/j.compedu.2018.10.008

22. Khribi MK, Jemni M, Nasraoui O (2008) Automatic Recommendations for E-Learning Personalization Based on Web Usage Mining Techniques and Information Retrieval. In: 2008 Eighth IEEE International Conference on Advanced Learning Technologies. IEEE, Santander, Cantabria, Spain. 241-245

23. Klašnja-Milićević A, Ivanović M, Vesin B, Budimac Z (2018) Enhancing e-learning systems with personalized recommendation based on collaborative tagging techniques. Appl Intell 48:1519-1535. https://doi.org/10.1007/s10489-017-1051-8

24. Kyaw Zaw S, Vasupongayya S (2019) A Case-Based Reasoning Approach for Automatic Adaptation of Classifiers in Mobile Phishing Detection. J Comp Networks Commun 2019:1-14. https://doi.org/10. $1155 / 2019 / 7198435$

25. Li Y, Xue F, Fan X et al (2018) Pedestrian walking safety system based on smartphone built-in sensors. IET Commun 12:751-758. https://doi.org/10.1049/iet-com.2017.0502

26. Li Y, Peng X, Zhou G, Zhao H (2020) SmartJump: A Continuous Jump Detection Framework on Smartphones. IEEE Internet Comput 24:18-26. https://doi.org/10.1109/MIC.2020.2969610

27. Li Y, Zhao X, Qu Z (2020) A Dynamic Programming Framework for Large-Scale Online Clustering on Graphs. Neural Process Lett 52:1613-1629. https://doi.org/10.1007/s11063-020-10329-1

28. Linder S, Whitehurst C (1973) Is there a Novelty Effect on Student Attitudes toward Personalized Instruction? J Exp Educ 42:42-44. https://doi.org/10.1080/00220973.1973.11011442

29. Oza KS, Naik PG (2016) Prediction of Online Lectures Popularity: A Text Mining Approach. Procedia Comp Sci 92:468-474. https://doi.org/10.1016/j.procs.2016.07.369

30. Palombi O, Jouanot F, Nziengam N et al (2019) OntoSIDES: Ontology-based student progress monitoring on the national evaluation system of French Medical Schools. Artif Intell Med 96:59-67. https:// doi.org/10.1016/j.artmed.2019.03.006

31. Pecori R (2018) A Virtual Learning Architecture Enhanced by Fog Computing and Big Data Streams. Future Internet 10:4. https://doi.org/10.3390/fi10010004 
32. Perron BE, Victor BG, Bushman G et al (2019) Detecting substance-related problems in narrative investigation summaries of child abuse and neglect using text mining and machine learning. Child Abuse Negl 98:104180. https://doi.org/10.1016/j.chiabu.2019.104180

33. Poppenk J, Köhler S, Moscovitch M (2010) Revisiting the novelty effect: When familiarity, not novelty, enhances memory. J Exp Psychol Learn Mem Cogn 36:1321-1330. https://doi.org/10.1037/a0019900

34. Qi J, Jiang G, Li G et al (2020) Surface EMG hand gesture recognition system based on PCA and GRNN. Neural Comput \& Applic 32:6343-6351. https://doi.org/10.1007/s00521-019-04142-8

35. Qiu L, Qi L (2019) E-learning assessment for tourism education LISREL assisted intercultural tourism perception and data integrated satisfaction perspectives. J Comput High Educ. https://doi.org/10.1007/ s12528-019-09223-0

36. Sarwar S, Qayyum ZU, García-Castro R et al (2019) Ontology based E-learning framework: A personalized, adaptive and context aware model. Multimed Tools Appl 78:34745-34771. https://doi.org/10. 1007/s 11042-019-08125-8

37. Setiawan AR (2020) Scientific Literacy Worksheets for Distance Learning in the Topic of Coronavirus 2019 (COVID-19)

38. Shahzad A, Hassan R, Aremu AY et al (2020) Effects of COVID-19 in E-learning on higher education institution students: the group comparison between male and female. Qual Quant. https://doi.org/10. $1007 / \mathrm{s} 11135-020-01028-\mathrm{Z}$

39. Sharif N, Afzal MT (2015) Recommendation approaches for e-learners: a survey. In: Proceedings of the 7th International Conference on Management of computational and collective intElligence in Digital EcoSystems - MEDES' '15. ACM Press, Caraguatatuba, Brazil. 137-141

40. Sheoran K, Tomar P, Mishra R (2020) A novel quality prediction model for component based software system using ACO-NM optimized extreme learning machine. Cogn Neurodyn 14:509-522. https://doi. org/10.1007/s11571-020-09585-7

41. Swadia J (2016) A study of text mining framework for automated classification of software requirements in enterprise systems

42. Tsay CH, Kofinas AK, Trivedi SK, Yang Y (2020) Overcoming the novelty effect in online gamified learning systems: An empirical evaluation of student engagement and performance. J Comput Assist Learn 36:128-146. https://doi.org/10.1111/jcal.12385

43. Viner RM, Russell SJ, Croker H et al (2020) School closure and management practices during coronavirus outbreaks including COVID-19: a rapid systematic review. Lancet Child Adoles Health 4:397404. https://doi.org/10.1016/S2352-4642(20)30095-X

Publisher's Note Springer Nature remains neutral with regard to jurisdictional claims in published maps and institutional affiliations.

\section{Authors and Affiliations}

\section{Sadia Ali ${ }^{1}$ - Yaser Hafeez ${ }^{1}$ - Muhammad Azeem Abbas ${ }^{1} \cdot$ Muhammad Aqib $^{1}$. Asif Nawaz ${ }^{1}$}

1 University Institute of Information Technology, PMAS Arid Agriculture University, Rawalpindi, Pakistan 